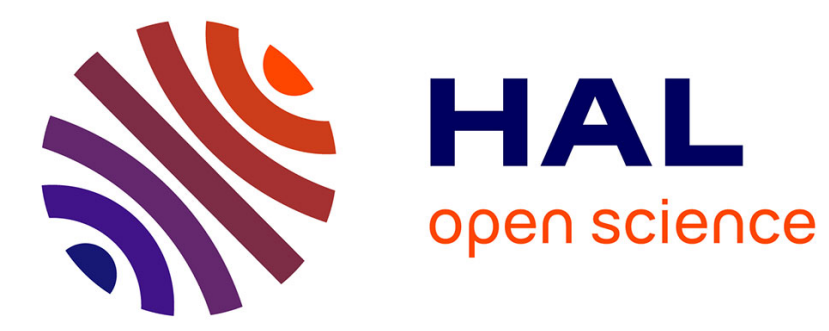

\title{
Fault-Tolerant Partial Replication in Large-Scale Database Systems
}

\author{
Pierre Sutra, Marc Shapiro
}

\section{To cite this version:}

Pierre Sutra, Marc Shapiro. Fault-Tolerant Partial Replication in Large-Scale Database Systems. [Research Report] RR-6440, INRIA. 2008, pp.25. inria-00232662v4

\section{HAL Id: inria-00232662 https://hal.inria.fr/inria-00232662v4}

Submitted on 31 Mar 2009

HAL is a multi-disciplinary open access archive for the deposit and dissemination of scientific research documents, whether they are published or not. The documents may come from teaching and research institutions in France or abroad, or from public or private research centers.
L'archive ouverte pluridisciplinaire HAL, est destinée au dépôt et à la diffusion de documents scientifiques de niveau recherche, publiés ou non, émanant des établissements d'enseignement et de recherche français ou étrangers, des laboratoires publics ou privés. 
INSTITUT NATIONAL DE RECHERCHE EN INFORMATIQUE ET EN AUTOMATIQUE

\title{
Fault-Tolerant Partial Replication in Large-Scale Database Systems
}

\author{
Pierre Sutra Marc Shapiro
}

Université Paris VI and INRIA Rocquencourt, France

$\mathbf{N}^{\circ} 6440$

Janvier 2008

Thème COM 



\title{
Fault-Tolerant Partial Replication in Large-Scale Database Systems
}

\author{
Pierre Sutra* Marc Shapiro \\ Université Paris VI and INRIA Rocquencourt, France \\ Thème COM — Systèmes communicants \\ Projet Regal \\ Rapport de recherche $\mathrm{n}^{\circ} 6440$ - Janvier 2008 - 22 pages
}

\begin{abstract}
We investigate a decentralised approach to committing transactions in a replicated database, under partial replication. Previous protocols either reexecute transactions entirely and/or compute a total order of transactions. In contrast, ours applies update values, and generate a partial order between mutually conflicting transactions only. It results that transactions execute faster, and distributed databases commit in small committees. Both effects contribute to preserve scalability as the number of databases and transactions increase. Our algorithm ensures serializability, and is live and safe in spite of faults.
\end{abstract}

Key-words: data replication, large-scale, database systems

* LIP6, 104, ave. du Président Kennedy, 75016 Paris, France; mailto:pierre.sutra@lip6.fr 
Un algoorithme tolérant aux fautes pour la réplication de bases de données dans les systèmes large-échelle Résumé :

Mots-clés : réplication, large-échelle, base de données, 


\section{Introduction}

Non-trivial consistency problems e.g. file systems, collaborative environments, and databases. are the major challenge of large-scale systems. Recently some architectures have emerged to scale file systems up to thousands of nodes [12, 15, 3, but no practical solution exists for database systems.

At the cluster level protocols based on group communication primitives [4, 11, 16] are the most promising solutions to replicate database systems [22]. In this article we extend the group communication approach to large-scale systems.

Highlights of our protocol:

- Replicas do not re-execute transactions, but apply update values only.

- We do not compute a total order over of operations. Instead transactions are partially ordered. Two transactions are ordered only over the data where they conflict.

- For every transaction $T$ we maintain the graph of $T$ 's dependencies. $T$ commits locally when $T$ is transitively closed in this graph.

The outline of the paper is the following. Section 2 introduces our model and assumptions. Section 3 presents our algorithm. We conclude in Section 4 after a survey of related work. An appendix follows containing a proof of correctness.

\section{System model and assumptions}

We consider a finite set of asynchronous processes or sites $\Pi$, forming a distributed system. Sites may fail by crashing, and links between sites are asynchronous but reliable. Each site holds a database that we model as some finite set of data items. We left unspecified the granularity of a data item. In the relational model, it can be a column, a table, or even a whole relational database. Given a data item $x$, the replicas of $x$, noted replicas $(x)$, are the subset of $\Pi$ whose databases contain $x$.

We base our algorithm on the three following primitives 1

- Uniform Reliable Multicast takes as input a unique message $m$ and a single group of sites $g \subseteq \Pi$. Uniform reliable multicast consists of the two primitives R-multicast $(m)$ and R-deliver $(m)$. With Uniform Reliable Multicast, all sites in $g$ have the following guarantees:

- Uniform Integrity: For every message $m$, every site in $g$ performs R-deliver $(m)$ at most once, and only if some site performed R-multicast $(m)$ previously.

- Validity: if a correct site in $g$ performs R-multicast $(m)$ then it eventually performs R-deliver $(m)$.

\footnotetext{
${ }^{1}$ Our taxonomy comes from [5].
} 


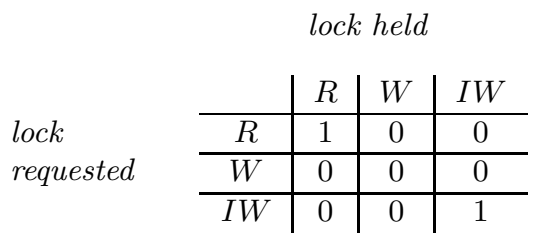

Table 1: Lock conflict table

- Uniform Agreement: if a site in $g$ performs R-deliver $(m)$, then every correct sites in $g$ eventually performs R-deliver $(m)$.

Uniform Reliable Multicast is solvable in an asynchronous systems with reliable links and crash-prone sites.

- Uniform Total Order Multicast takes as input a unique message $m$ and a single group of sites $g$. Uniform Total Order Multicast consists of the two primitives TO-multicast $(m)$ and TO-deliver $(m)$. This communication primitive ensures Uniform Integrity, Validity, Uniform Agreement and Uniform Total Order in $g$ :

- Uniform Total Order: if a site in $g$ performs TO-deliver $(m)$ and TO-deliver $\left(m^{\prime}\right)$ in this order, then every site in $g$ that performs TO-deliver $\left(m^{\prime}\right)$ has performed previously TO-deliver $(m)$.

- Eventual Weak Leader Service Given a group of sites $g$, a site $i \in g$ may call function WLeader $(g)$. WLeader $(g)$ returns a weak leader of $g$ :

- WLeader $(g) \in g$.

- Let $\rho$ be a run of $\Pi$ such that a non-empty subset $c$ of $g$ is correct in $\rho$. It exists a site $i \in c$ and a time $t$ such that for any calls of $W \operatorname{Leader}(g)$ on $i$ after $t$, $W L e a d e r(g)$ returns $i$.

This service is strictly weaker than the classical eventual leader service $\Omega$ [18, since we do not require that every correct site eventually outputs the same leader. An algorithm that returns to every process itself, trivially implements the Eventual Weak Leader Service.

In the following we make two assumptions: during any run, (A1) for any data item $x$, at least one replica of $x$ is correct, and (A2) Uniform Total Order Multicast is solvable in replicas $(x)$.

\subsection{Operations and locks}

Clients of the system (not modeled), access data items using read and write operations. Each operation is uniquely identified, and accesses a single data item. A read operation is 
a singleton: the data item read, a write operation is a couple: the data item written, and the update value.

When an operation accesses a data item on a site, it takes a lock. We consider the three following types of locks: read lock $(\mathrm{R})$, write lock $(\mathrm{W})$, and intention to write lock (IW).Table 1 illustrates how locks conflict with each other; when an operation requests a lock to access a data item, if the lock is already taken and cannot be shared, the request is enqueued in a FIFO queue. In Table 1, 0 means that the request is enqueued, and 1 that the lock is granted.

Given an operation $o$, we note:

- item(o), the data item operation $o$ accesses,

- isRead(o) (resp. isWrite(o)) a boolean indicating whether $o$ is a read (resp. a write),

- and $\operatorname{replicas}(o) \triangleq \operatorname{replicas}(\operatorname{item}(o))$;

We say that two operations $o$ and $o^{\prime}$ conflict if they access the same data item and one of them is a write:

$$
\operatorname{conflict}\left(o, o^{\prime}\right) \triangleq\left\{\begin{array}{l}
\text { item }(o)=\operatorname{item}\left(o^{\prime}\right) \\
\text { isWrite }(o) \vee \text { is Write }\left(o^{\prime}\right)
\end{array}\right.
$$

\section{$2.2 \quad$ Transactions}

Clients group their operations into transactions. A transaction is a uniquely identified set of read and write operations. Given a transaction $T$,

- for any operation $o \in T$, function $\operatorname{trans}(o)$ returns $T$,

- $r o(T)$ (respectively $w o(T)$ ) is the subset of read (resp. write) operations,

- $\operatorname{item}(T)$ is the set of data items transaction $T$ accesses: item $(T) \triangleq \bigcup_{o \in T}$ item $(o)$.

- and $\operatorname{replicas}(T) \triangleq \operatorname{replicas}(\operatorname{item}(T))$.

Once a site $i$ grants a lock to a transaction $T, T$ holds it until $i$ commits $T, i$ aborts $T$, or we explicitly say that this lock is released.

\section{The algorithm}

As replicas execute transactions, it creates precedence constraints between conflicting transactions. Serializability theory tell us that this relation must be acyclic [2].

One solution to this problem, is given a transaction $T$, (i) to execute $T$ on every replicas of $T$, (ii) to compute the transitive closure of the precedence constraints linking $T$ to concurrent conflicting transactions, and (iii) if a cycle appears, to abort at least one the transactions involved in this cycle. 
Unfortunately as the number of replicas grows, sites may crash, and the network may experience congestion. Consequently to compute (ii) the replicas of $T$ need to agree upon the set of concurrent transactions accessing item $(T)$.

Our solution is to use a TO-multicast protocol per data item.

\section{$3.1 \quad$ Overview}

To ease our presentation we consider in the following that a transaction executes initially on a single site. Section 3.9 generalizes our approach to the case where a transaction initially executes on more than one site. We structure our algorithm in five phases:

- In the initial execution phase, a transaction $T$ executes at some site $i$.

- In the submission phase, $i$ transmits $T$ to replicas $(T)$.

- In the certification phase, a site $j$ aborts $T$ if $T$ has read an outdated value. If $T$ is not aborted, $j$ computes all the precedence constraints linking $T$ to transactions previously received at site $j$.

- In the closure phase, $j$ completes its knowledge about precedence constraints linking $T$ to others transactions.

- Once $T$ is closed at site $j$, the commitment phase takes place. $j$ decides locally whether to commit or abort $T$. This decision is deterministic, and identical on every site replicating a data item written by $T$.

\subsection{Initial execution phase}

A site $i$ executes a transaction $T$ coming from a client according to the two-phases locking rule 2, but without applying write operation: 2 . When site $T$ reaches a commit statement, it is not committed, instead $i$ releases $T$ 's read locks, converts $T$ 's write locks into intention to write locks, computes T's update values, and then proceeds to the submission phase.

\subsection{Submission phase}

In this phase $i$ R-multicasts $T$ to replicas $(T)$. When a site $j$ receives $T, j$ marks all $T$ 's operations as pending using variable pending. Then if it exists an operation $o \in$ pending, such that $j=W$ Leader $($ replicas $(o)), j$ TO-multicasts $o$ to replicas $(o) 3$

\footnotetext{
${ }^{2}$ If $T$ writes a data item $x$ then reads it, we suppose some internals to ensure that $T$ sees a consistent value.

${ }^{3}$ If instead of this procedure, $i$ TO-multicasts all the operations, then the system blocks if $i$ crashes. We use a weak leader and a reliable multicast to preserve liveness.
} 


\subsection{Certification phase}

When a site $i$ TO-delivers an operation o for the first tim $4, i$ removes $o$ from pending, $i$ certifies $o$.

To certify $o, i$ considers any preceding write operations that conflicts with $o$. We say that a conflicting operation $o^{\prime}$ precedes o at site $i, o^{\prime} \rightarrow_{i} o$, if $i$ TO-delivers $o^{\prime}$ then $i$ TO-delivers $o$ :

$$
o^{\prime} \rightarrow_{i} o \triangleq\left\{\begin{array}{l}
\text { TO-deliver }_{i}\left(o^{\prime}\right) \prec \text { TO-deliver }_{i}(o) \\
\operatorname{conflict}\left(o^{\prime}, o\right)
\end{array}\right.
$$

Where given two events $e$ and $e^{\prime}, e \prec e^{\prime}$ is the relation e happens-before $e^{\prime}$, and TO-deliver ${ }_{i}\left(o^{\prime}\right)$ is the event: "site $i$ TO-delivers operation $o^{\prime}$ ".

If $o$ is a read, we check that $o$ did not read an outdated value. It happens when $o$ executes concurrently to a conflicting write operation $o^{\prime}$ that is now committed. Let committed $d_{i}$ be the set of transactions committed at site $i$, the read operation $o$ aborts, if it exists an operation $o^{\prime}$ such that $o^{\prime} \rightarrow_{i} O \wedge \operatorname{trans}\left(o^{\prime}\right) \| \operatorname{trans}(o) \wedge \operatorname{trans}\left(o^{\prime}\right) \in$ committed $_{i}$, where $\operatorname{trans}\left(o^{\prime}\right) \| \operatorname{trans}(o)$ means that the transactions $\operatorname{trans}\left(o^{\prime}\right)$ and $\operatorname{trans}(o)$ were executed concurrently during the initial execution phase.

If now $o$ is a write, $i$ gives an IW lock to $o$ : function forceWriteLock $(o)$. If an operation $o^{\prime}$ holds a conflicting IW lock, $o$ and $o^{\prime}$ share the lock (see Table 1); otherwise it means that $\operatorname{trans}\left(o^{\prime}\right)$ is still executing at site $i$, and function forceWriteLock(o) aborts it 5

\subsection{Precedence graph}

Our algorithm decides to commit or abort transactions, according to a precedence graph. A precedence graph $G$ is a directed graph where each node is a transaction $T$, and each directed edge $T \rightarrow T^{\prime}$, models a precedence constraint between an operation of $T$, and a write operation of $T^{\prime}$ :

$$
T \rightarrow T^{\prime} \triangleq \exists\left(o, o^{\prime}\right) \in T \times T^{\prime}, \exists i \in \Pi, o^{\prime} \rightarrow_{i} O
$$

A precedence graph contains also for each vertex $T$ a flag indicating whether $T$ is aborted or not: isAborted $(T, G)$, and the subset of $T$ 's operations: $o p(T, G)$, which contribute to the relations linking $T$ to others transactions in $G$.

Given a precedence graph $G$, we note $G \cdot \mathcal{V}$ its vertices set, and $G \cdot \mathcal{E}$ its edges set. Let $G$ and $G^{\prime}$ be two precedence graphs, the union between $G$ and $G^{\prime}, G \cup G^{\prime}$, is such that:

. $\left(G \cup G^{\prime}\right) \cdot \mathcal{V}=G \cdot \mathcal{V} \cup G^{\prime} \cdot \mathcal{V}$,

- $\left(G \cup G^{\prime}\right) \cdot \mathcal{E}=G \cdot \mathcal{E} \cup G^{\prime} \cdot \mathcal{E}$,

- $\forall T \in\left(G \cup G^{\prime}\right) . \mathcal{V}$, isAborted $\left(T,\left(G \cup G^{\prime}\right)\right)=\operatorname{isAborted}(T, G) \vee \operatorname{isAborted}\left(T, G^{\prime}\right)$.

- $\forall T \in\left(G \cup G^{\prime}\right) . \mathcal{V}$, op $\left(T,\left(G \cup G^{\prime}\right)\right)=o p(T, G) \cup$ op $\left(T, G^{\prime}\right)$.

\footnotetext{
${ }^{4}$ Recall that the leader is eventual, consequently $i$ may receive $o$ more than one time.

${ }^{5}$ This operation prevents local deadlocks.
} 


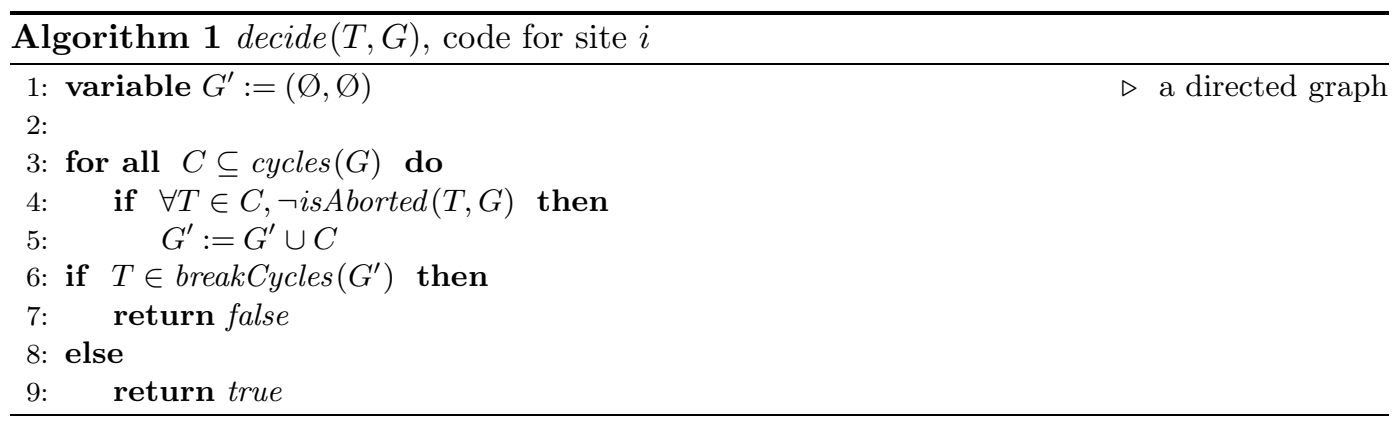

We say that $G$ is a subset of $G^{\prime}$, noted $G \subseteq G^{\prime}$, if:

. $G . \mathcal{V} \subseteq G^{\prime} . \mathcal{V} \wedge G . \mathcal{E} \subseteq G^{\prime} \cdot \mathcal{E}$,

- $\forall T \in G . \mathcal{V}$, isAborted $(T, G) \Rightarrow \operatorname{isAborted}\left(T, G^{\prime}\right)$,

- $\forall T \in G$. $\mathcal{V}, o p(T, G) \subseteq o p\left(T, G^{\prime}\right)$.

Let $G$ be a precedence graph, $\operatorname{in}(T, G)$ (respectively out $(T, G)$ ) is the restriction of $G . \mathcal{V}$ to the subset of vertices formed by $T$ and its incoming (resp. outgoing) neighbors. The predecessors of $T$ in $G: \operatorname{pred}(T, G)$, is the precedence graph representing the transitive closure of the dual of the relation $G \cdot \mathcal{E}$ on $\{T\}$.

\subsection{Deciding}

Each site $i$ stores its own precedence graph $G_{i}$, and decides locally to commit or abort a transaction according to it. More precisely $i$ decides according to the $\operatorname{graph} \operatorname{pred}\left(T, G_{i}\right)$. For any cycle $C$ in the set of cycles in $\operatorname{pred}\left(T, G_{i}\right): \operatorname{cycles}\left(\operatorname{pred}\left(T, G_{i}\right)\right)$. $i$ must abort at least one transaction in $C$. This decision is deterministic, and $i$ tries to minimize the number of transactions aborted.

Formally speaking $i$ solves the minimum feedback vertex set problem over the union of all cycles in $\operatorname{pred}\left(T, G_{i}\right)$ containing only non-aborted transactions The minimum feedback vertex set problem is an NP-complete optimization problem, and the literature about this problem is vast 6 . We consequently postulate the existence of an heuristic: breakCycles(). breakCycles() takes as input a directed graph $G$, and returns a vertex set $S$ such that $G \backslash S$ is acyclic.

Now considering a transaction $T \in G_{i}$ such that $G=\operatorname{pred}\left(T, G_{i}\right)$, Algorithm 1 returns false if $i$ aborts $T$, or true otherwise.

\subsection{Closure phase}

In our model sites replicate data partially, and consequently maintain an incomplete view of the precedence constraints linking transactions in the system. Consequently they need to complete their view by exchanging parts of their graphs. This is our closure phase: 
- When $i$ TO-delivers an operation $o \in T, i$ adds $T$ to its precedence graph, and adds $o$ to op $\left(T, G_{i}\right)$. Then $i$ sends $\operatorname{pred}\left(T, G_{i}\right)$ to replicas $\left(\right.$ out $\left(T, G_{i}\right)$ ) (line 29).

- When $i$ receives a precedence graph $G$, if $G \nsubseteq G_{i}$, for every transaction $T$ in $G_{i}$, such that $\operatorname{pred}(T, G) \nsubseteq \operatorname{pred}\left(T, G_{i}\right), i$ sends $\operatorname{pred}\left(T, G \cup G_{i}\right)$ to $\operatorname{replicas}\left(\operatorname{out}\left(T, G_{i}\right)\right)$. Then $i$ merges $G$ to $G_{i}$ (lines 31 to 35 ).

Once $i$ knows all the precedence constraints linking $T$ to others transactions, we say that $T$ is closed at site $i$. Formally $T$ is closed at site $i$ when the following fixed-point equation is true at site $i$ :

$$
\operatorname{closed}\left(T, G_{i}\right)=\left\{\begin{array}{l}
o p(T, G)=T \\
\forall T^{\prime} \in \operatorname{in}\left(T, G_{i}\right) \cdot \mathcal{V}, \operatorname{closed}\left(T^{\prime}, G_{i}\right)
\end{array}\right.
$$

Our closure phase ensures that during every run $\rho$, for every correct site $i$, and every transaction $T$ which is eventually in $G_{i}, T$ is eventually closed at site $i$.

\subsection{Commitment phase}

If $T$ is a read-only transaction: $w o(T)=\varnothing, i$ commits $T$ as soon as $T$ is executed (line 9).

If $T$ is an update, $i$ waits that $T$ is closed and holds all its IW locks: function holdIWLocks() (line 35). Once these two conditions hold, $i$ computes decide $\left(T, \operatorname{pred}\left(T, G_{i}\right)\right)$. If this call returns true, $i$ commits $T$ : for each write operation $o \in w o(T)$, with $i \in$ replicas $(o), i$ considers any write operation $o^{\prime}$ such that $T \rightarrow \operatorname{trans}\left(o^{\prime}\right) \in G_{i} \wedge \operatorname{conflict}\left(o, o^{\prime}\right)$. If $\operatorname{trans}\left(o^{\prime}\right)$ is already committed at site $i, i$ does nothing; otherwise $i$ applies $o$ to its database.

Algorithm 2 describes our algorithm. This protocol provides serializability for partially replicated database systems: any run of this protocol is equivalent to a run on a single site [2]. The proof of correctness appears in Appendix.

\subsection{Initial execution on more than one site}

When initial execution phase does not take place on a single site we compute the read-from dependencies. More precisely when a site $i$ receives a read $o$ accessing a data item it does not replicate, $i$ sends $o$ to some replica $j \in$ replicas $(o)$. Upon reception $j$ executes $o$. At the end of execution $j$ sends back to $i$ the transitive closure containing read-from dependencies and starting from $T$.

Once $i$ has executed locally or remotely all the read operations in $T, i$ checks if the resulting graph contains cycles in which $T$ is involved. If this is the case, $T$ will be aborted, and instead of submitting it, $i$ re-executes at least one of $T$ 's read operations Otherwise $i$ computes the write set and the update values, and sends $T$ with its read-from dependencies by Uniform Reliable Multicast. The dependencies are merged to precedence graph when a site receives an operation by Total Order Multicast. The rest of the algorithm remains the same. 


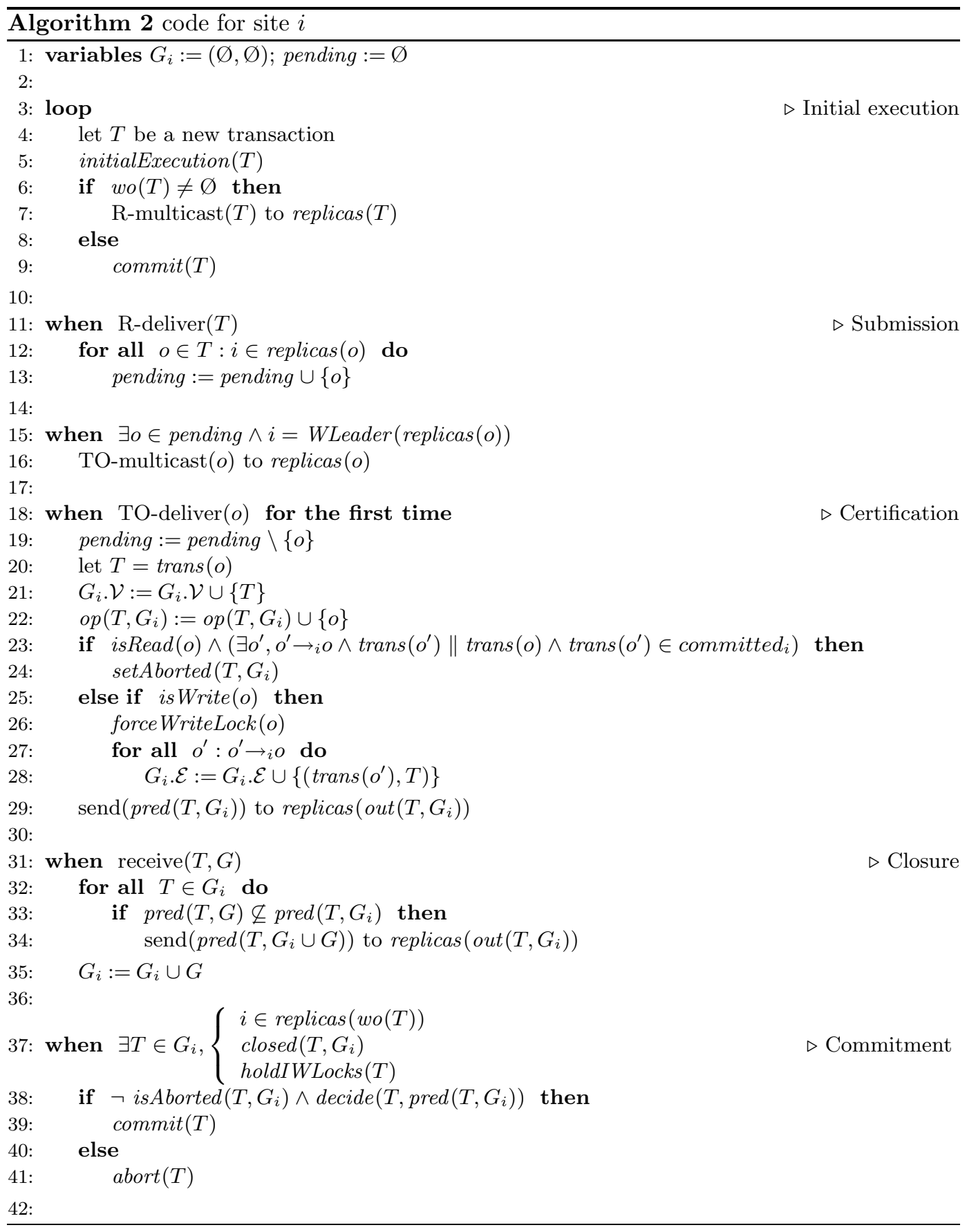




\subsection{Performance analysis}

We consider Paxos 14 as a solution to Uniform Total Order Multicast. Since precedence constraints in a cycle are not causally related, Algorithm 2 achieves a message delay of 5: 2 for Uniform Reliable Multicast, and 3 for Uniform Total Order Multicast. It reduces to 4, if in each replica group the leader of Paxos is also the weak leader of $g$.

Let $o$ be the number of operations per transaction, and $d$ be the replication degree, the message complexity of Algorithm 2 is $5 o d+(o d)^{2}$ : $2 o d$ for Uniform Reliable Multicast, o Uniform Total Order Multicasts, each costing $2 d$ messages, and od replicas execute line 29. each site sending od messages. Again, if in each replica group, the leader of Paxos is also the weak leader of $g$, the message complexity of our protocol reduces to $4 o d+(o d)^{2}$

\section{Concluding remarks}

\subsection{Related work}

Gray et al. 7] prove that scale traditional eager and lazy replications does not scale: the deadlock rate increase as the cube of the number of sites, and the reconciliation rate increases as the square. Wiesmann and Schiper confirm practically this result [22]. Fritzke et al. [10] propose a replication scheme where sites TO-multicast each operations and execute them upon reception. However they do not prevent global deadlocks with a priority rule; it increases abort rate. Preventive replication [16] considers that a bound on processor speed, and network delay is known. Such assumptions do not hold in a large-scale system. The epidemic algorithm of Holiday et al [9] aborts concurrent conflicting transactions and their protocol is not live in spite of one fault. In all of these replication schemes, each replica execute all the operations accessing the data items it replicates. Alonso proves analytically that it reduces the scale-up of the system 11 .

The DataBase State Machine approach [17] applies update values only but in a fully replicated environment. Its extensions [19, 21] to partial replication require a total order over transactions.

Committing transactions using a distributed serialization graph is a well-known technique [20. Recently Haller et al. have proposed to apply it [8 to large-scale systems, but their solution does not handle replication, nor faults.

\subsection{Conclusion}

We present an algorithm for replicating database systems in a large-scale system. Our solution is live and safe in presence of non-byzantine faults. Our key idea is to order conflicting transaction per data item, then to break cycles between transactions. Compared to previous existing solutions, ours either achieves lower latency and message cost, or does not unnecessarily abort concurrent conflicting transactions.

The closure of constraints graphs is a classical idea in distributed systems. We may find it in the very first algorithm about State Machine Replication [13], or in a well-known 
algorithm to solve Total Order Multicast [5] 6 We believe that the closure generalizes to a wider context, where a constraint is a temporal logic formula over sequences of concurrent operations.

\section{References}

[1] G. Alonso. Partial database replication and group communication primitives in 2nd European Research Seminar on Advances in Distributed Systems, 1997.

[2] Philip A. Bernstein, Vassos Hadzilacos, and Nathan Goodman. Concurrency Control and Recovery in Database Systems. Addison-Wesley, 1987.

[3] J-M. Busca, F. Picconi, and P. Sens. Pastis: A highly-scalable multi-user peer-to-peer file system. In Euro-Par, 2005.

[4] L. Camargos, F. Pedone, and M. Wieloch. Sprint: a middleware for high-performance transaction processing. SIGOPS Oper. Syst. Rev., 2007.

[5] X. Defago, A. Schiper, and P. Urban. Totally ordered broadcast and multicast algorithms: a comprehensive survey, 2000.

[6] Michael R. Garey and David S. Johnson. Computers and Intractability; A Guide to the Theory of NP-Completeness. W. H. Freeman \& Co., New York, NY, USA, 1990.

[7] J. Gray, P. Helland, P. O'Neil, and D. Shasha. The dangers of replication and a solution. In Proceedings of the 1996 ACM SIGMOD international conference on Management of data, 1996.

[8] K. Haller, H. Schuldt, and C. Türker. Decentralized coordination of transactional processes in peer-topeer environments. In CIKM '05: Proceedings of the 14th ACM international conference on Information and knowledge management, 2005.

[9] J. Holliday, D. Agrawal, and A. Abbadi. Partial database replication using epidemic communication, 2002.

[10] U. Fritzke Jr. and P. Ingels. Transactions on partially replicated data based on reliable and atomic multicasts. In Proceedings of the The 21st International Conference on Distributed Computing Systems, page 284. IEEE Computer Society, 2001.

[11] Bettina Kemme and Gustavo Alonso. Don't be lazy, be consistent: Postgres-r, a new way to implement database replication. In The VLDB Journal, pages 134-143, 2000.

[12] J. Kubiatowicz, D. Bindel, Y. Chen, P. Eaton, D. Geels, R. Gummadi, S. Rhea, H. Weatherspoon, W. Weimer, C. Wells, and B. Zhao. Oceanstore: An architecture for global-scale persistent storage. In Proceedings of ACM ASPLOS. ACM, November 2000.

[13] Leslie Lamport. Time, clocks, and the ordering of events in a distributed system. Commun. ACM, 21(7):558-565, 1978.

[14] Leslie Lamport. Fast paxos. Distributed Computing, 19(2):79-103, October 2006.

[15] A. Muthitacharoen, R. Morris, T. M. Gil, and B. Chen. Ivy: A read/write peer-to-peer file system. In Proceedings of 5th Symposium on Operating Systems Design and Implementation, 2002.

[16] Esther Pacitti, Cédric Coulon, Patrick Valduriez, and M. Tamer Özsu. Preventive replication in a database cluster. Distrib. Parallel Databases, 18(3):223-251, 2005.

[17] F Pedone, R Guerraoui, and A Schiper. The database state machine approach. Distrib. Parallel Databases, 14(1):71-98, July 2003.

\footnotetext{
${ }^{6}$ In [13] Lamport closes the $\ll$ relation for every request to the critical section. In [5] the total order multicast protocol attributed to Skeen, closes the order over natural numbers to TO-multicast a message.
} 
[18] Michel Raynal. Eventual leader service in unreliable asynchronous systems: Why? how? In $N C A$, pages 11-24. IEEE Computer Society, 2007.

[19] N. Schiper, R. Schmidt, and F. Pedone. Optimistic algorithms for partial database replication. In 10th International Conference on Principles of Distributed Systems (OPODIS'2006), 2006.

[20] C-S Shih and J. A. Stankovic. Survey of deadlock detection in distributed concurrent programming environments and its application to real-time systems. Technical report, 1990.

[21] A. Sousa, F. Pedone, R. Oliveira, and F. Moura. Partial replication in the database state machine, 2001.

[22] M. Wiesmann and A. Schiper. Comparison of database replication techniques based on total order broadcast. IEEE Transactions on Knowledge and Data Engineering, 17(4), 2005. 


\section{$.1 \quad$ Additionnal notations}

We note $\mathbb{D}$ the universal set of data item, $\mathbb{T}$ the universal set of transactions, and $\mathbb{G}$ the universal set of precedence graphs constructed upon $\mathbb{D}$.

Let $\rho$ be a run of Algorithm2, given a site $i$ we note event $t_{i}$ when the event event happens at site $i$ during $\rho$; moreover if value is the result of this event we note it: event $t_{i}=$ value.

Let $\rho$ be a run of Algorithm 2, we note:

- $\operatorname{faulty}(\rho)$ the set of sites that crashes during $\rho$,

- $\operatorname{correct}(\rho)$ the set $\Pi \backslash \operatorname{faulty}(\rho)$.

- $\operatorname{committed}(\rho)$ the transactions committed during $\rho$, i.e. $\left\{T \in \mathbb{T}, \exists i \in \Pi, T \in\right.$ committed $\left._{i}\right\}$,

- and aborted $(\rho)$ the transactions aborted during $\rho$, i.e. $\left\{T \in \mathbb{T}, \exists i \in \Pi, T \in\right.$ aborted $\left._{i}\right\}$.

Given a site $i$ and a time $t$, we note $G_{i, t}$ the value of $G_{i}$ at time $t$.

\section{$.2 \quad$ Proof of correctness}

Since the serializability theory is over a finite set of transactions, we suppose hereafter that during $\rho$ a finite subset of $\mathbb{T}$ is sent to the system.

Let $\rho$ be a run of Algorithm 2, we now proove a series of propositions leading to the fact that $\rho$ is serializable.

\section{P1}

$\forall T \in \mathbb{T},\left(\exists j \in \Pi, \mathrm{R}_{\text {-deliver }}(T) \in \rho\right)$

$$
\Rightarrow\left(\forall o \in T, \forall i \in \operatorname{replicas}(o) \cap \operatorname{correct}(\rho), \operatorname{TO}^{\left.-\operatorname{deliver}_{i}(o) \in \rho\right)}\right.
$$

Proof

Let $T$ be a transaction and $j$ a site that R-delivers $T$ during $\rho$.

F1.1 $\forall i \in \operatorname{replicas}(T) \cap \operatorname{correct}(\rho), \mathrm{R}_{-\operatorname{deliver}_{i}}(T)$

By the Uniform Agreement property of Uniform Reliable Multicast.

F1.2 $\forall o \in T, \exists k \in \operatorname{correct}(\rho) \cap \operatorname{replicas}(o)$, TO-multicast $_{k}(o) \in \rho$

F1.2.1 $\exists l \in \operatorname{correct}(\rho) \cap$ replicas $(o)$, WLeader $_{l}\left(\right.$ replicas $\left.\left._{(o)}\right)\right)=l \wedge \mathrm{R}_{\text {-deliver }}(o)$

By fact F.1.1, assumption A1 and the properties of the Eventual Weak Leader Service.

By fact F1.2.1 eventually a correct site executes line 16 in Algorithm 2. 
Fact F1.2 and the Validity and the Agreement properties of Total Order Multicast conclude our claim.

In the following we say that a transaction $T$ is submitted to the system: $T \in$ submitted $(\rho)$, if a site i R-delivers $T$ during $\rho$.

\section{P2}

$\forall T \in \operatorname{submitted}(\rho), \forall i \in \operatorname{replicas}(T), \forall o \in T$

$$
\exists G \in \mathbb{G}, o \in \text { op }(T, G) \wedge \operatorname{receive}_{i}(G)
$$

Proof

F2.1 $\forall i \in \Pi, \forall t, t^{\prime}, t>t^{\prime} \Rightarrow G_{i, t} \subseteq G_{i, t^{\prime}}$

F2.2 $\forall G \in \mathbb{G}, \forall T \in \mathbb{T}, T \in G \Rightarrow T \in \operatorname{pred}(T, G)$

By definition of $\operatorname{pred}(T, G)$.

By proposition P1, facts F2.1 and F2.2, and since links are reliable.

\section{P3}

$\forall T, T^{\prime} \in \operatorname{submitted}(\rho)$,

$$
T \rightarrow T^{\prime} \Rightarrow\left(\exists o, o^{\prime} \in T \times T^{\prime}, \exists i \in \operatorname{correct}(\rho), o \rightarrow{ }_{i} o^{\prime}\right)
$$

Proof

By definition of $T \rightarrow T^{\prime}$, let $o, o^{\prime} \in T \times T^{\prime}$ and let $j$ be a site such that $o \rightarrow_{j} o^{\prime}$. Since conflict $\left(o, o^{\prime}\right)$ and an operation applies on a single data item, we note $x$ the unique data item such that $x=\operatorname{item}(o)=\operatorname{item}\left(o^{\prime}\right)$.

F3.1 $j \in$ replicas $(x)$

Site $j$ TO-delivers $o$ during $\rho$ and links are reliable.

F3.2 $\exists i \in \operatorname{replicas}(x) \cap \operatorname{correct}(\rho)$, TO-deliver $_{i}(o) \wedge$ TO-deliver ${ }_{i}\left(o^{\prime}\right)$ 
By assumption A1 $\exists i \in \operatorname{replicas}(x) \cap \operatorname{correct}(\rho)$, and by the Uniform Agreement property of Total Order Multicast, since $i$ is correct during $\rho, i$ TO-delivers both $o$ and $o^{\prime}$.

Fact F3.2 and the Total Order property of Total Order Multicast concludes our claim.

\section{$\mathbf{P 4}$}

$\forall T \in \operatorname{submitted}(\rho), \forall i \in \Pi$

$\left(\exists t, T \in G_{i, t}\right) \Rightarrow\left(\exists T_{1}, \ldots, T_{m \geq 0} \in \operatorname{submitted}(\rho), i \in \operatorname{replicas}\left(T_{m}\right) \wedge T \rightarrow T_{1} \rightarrow \ldots \rightarrow T_{m}\right)$

Proof

Since $G_{i, 0}=(\varnothing, \varnothing)$, let us consider the first time $t_{0}$ at which $T \in G_{i, t}$.

According to Algorithm 2 either:

- $i$ TO-delivers an operation $o \in T$ at $t_{0}$, and thus $i \in \operatorname{replicas}(T)$. QED

- or $i$ receives a precedence graph $G^{\prime}$ from a site $j$ such that $T \in G^{\prime}$. Now since links are reliable, note $t_{1}$ the time at which $j$ send $G^{\prime}$ to $i$. According to lines 29 and 34 it exists a transactions $T^{\prime}$ such that $T \in \operatorname{pred}\left(T^{\prime}, G_{j, t_{1}}\right)$, and a transaction $T^{\prime \prime}$ such that $T^{\prime \prime} \in \operatorname{out}\left(T^{\prime}, G_{j, t_{1}}\right)$ and $i \in \operatorname{replicas}\left(T^{\prime \prime}\right)$.

From $T \in \operatorname{pred}\left(T^{\prime},\right)$, by definition of the predecessors, we obtain $T \rightarrow \ldots \rightarrow T^{\prime}$, and from $T^{\prime \prime} \in \operatorname{out}\left(T^{\prime}, G_{j, t_{1}}\right)$ we obtain $T^{\prime} \rightarrow T^{\prime \prime}$. Thus $T \rightarrow \ldots \rightarrow T^{\prime} \rightarrow T^{\prime \prime}$, with $i \in \operatorname{replicas}\left(T^{\prime \prime}\right)$.

\section{P5}

$\forall T \in \operatorname{submitted}(\rho), \forall i \in \operatorname{correct}(\rho)$,

$$
\left(\exists t, T \in G_{i, t}\right) \Rightarrow\left(\exists t, o p\left(T, G_{i, t}\right)=T\right)
$$

Proof

Let $T_{0}$ be a transaction submitted during $\rho$ and let $i$ be a site that eventually hold $T_{0}$ in $G_{i}$.

By proposition $\mathbf{P} 4$ it exists $T_{1}, \ldots, T_{m \geq 0} \in \operatorname{submitted}(\rho)$ such that $i \in \operatorname{replicas} T_{m}$ and $T \rightarrow T_{1} \rightarrow \ldots \rightarrow T_{m}$.

Let $k \in \llbracket 0, m \rrbracket$, we note $\mathcal{P}(k)$ the following property: 


$$
\mathcal{P}(k) \triangleq \forall j \in \operatorname{correct}(\rho) \cap \operatorname{replicas}\left(T_{k}\right), \exists t \in o p\left(T_{0}, G_{j, t_{0}}\right)=T_{0}
$$

Observe that by proposition $\mathbf{P} 2 \mathcal{P}(0)$ is true. We now proove that $\mathcal{P}(k)$ is true for all the $k$ by induction:

Let $o, o^{\prime} \in T_{k} \times T_{k+1}$, and $j \in \operatorname{correct}(\rho)$ such that $o \rightarrow{ }_{j} o^{\prime}$.

Let $t_{0}$ be the first time at which $j$ TO-delivers $o$ during $\rho$. true).

Let $t_{2}$ be the first time at which op $\left(T_{k}, G_{j, t}\right)=T_{k}$ ( since $G_{j, 0}=(\varnothing, \varnothing)$, and $\mathcal{P}(k)$ is

Let $t_{1}$ be the first time at which $j$ To-delivers $o^{\prime}$ during $\rho$.

Observe that since $o \rightarrow_{j} o^{\prime}, t_{O}<t_{1}$. It follow that we have three cases to consider:

- cases $t_{2}<t_{0}<t_{1}$ and $t_{0}<t_{2}<t_{1}$

In these cases when $j$ To-delivers $o^{\prime}$, we have:

$$
T_{k} \rightarrow T_{k+1} \in G_{j, t_{1}} \wedge o p\left(T_{k}, G_{j, t_{1}}=T_{k}\right)
$$

Thus,

$$
T_{k} \in \operatorname{pred}\left(T_{k+1}, G_{j, t_{1}}\right) \wedge o p\left(T_{k}, \operatorname{pred}\left(T_{k}, G_{j, t_{1}}\right)\right)=T_{k}
$$

and according to Algorithm 2, $j$ sends $\operatorname{pred}\left(T_{k+1}, G_{j, t_{1}}\right)$ to $\operatorname{replicas}\left(\operatorname{out}\left(T_{k+1},\right)\right) G_{j, t_{1}}$. Now since replicas $\left(T_{k+1}\right) \subseteq \operatorname{replicas}\left(\operatorname{out}\left(T_{k+1},\right)\right) G_{j, t_{1}}$, given a site $j \in \operatorname{replicas}\left(T_{k+1}\right)$, eventually $j$ receives $\operatorname{pred}\left(T_{k+1}, G_{j, t_{1}}\right)$, and merges it into its own precedence graph.

- case $t_{0}<t_{1}<t_{2}$

We consider two-subcases:

- At $t_{2} j$ delivers an operation of $T_{k}$, and this operation is different from $o^{\prime}$. Now since $T_{k} \rightarrow T_{k+1} \in G_{j, t_{2}}, \mathcal{P}(k+1)$ is true.

- If now $j$ receives a graph $G$ such that $o p\left(T_{k}, G\right)=T_{k}$, by definition of $t_{2}, G \subseteq$ $G_{j, t_{2}}$, and more precisely, $\operatorname{pred}\left(T_{k}, G\right) \nsubseteq \operatorname{pred}\left(T_{k}, G_{j, t_{2}}\right)$.

It follows that $j$ sends $\operatorname{pred}\left(T_{k}, G \cup G_{j, t_{2}}\right)$ to replicas $\left(\operatorname{out}\left(T_{k}, G \cup \cup G_{j, t_{2}}\right)\right)$. Finally since by definition of $t_{1}, T_{k} \rightarrow T_{k+1} \in G_{j, t_{2}}$, we obtain $T_{k+1} \in \operatorname{out}\left(T_{k}, G \cup G_{j, t_{2}}\right)$, from which we conclude that $\mathcal{P}(k+1)$ is true. $T_{0}$.

To conclude observe that since $i \in \operatorname{replicas}\left(T_{m}\right)$ and $\mathcal{P}(m)$ is true, eventually op $\left(T_{0}, G_{i, t_{0}}\right)=$ 


\section{P6}

$\forall T \in \operatorname{submitted}(\rho), \forall i \in \operatorname{correct}(\rho)$,

$$
\left(\exists t, T \in G_{i, t}\right) \Rightarrow\left(\exists t, \forall T^{\prime} \in \operatorname{submitted}(\rho), T^{\prime} \rightarrow T \Rightarrow\left(T^{\prime}, T\right) \in G_{i, t}\right)
$$

Proof

F6.1 $\forall T, T^{\prime} \in$ submitted $\rho, \forall o, o^{\prime} \in T \times T^{\prime},\left(\exists i \in \Pi, o^{\prime} \rightarrow \Rightarrow o \forall j \in \operatorname{replicas}(o), o \rightarrow{ }_{j} o^{\prime}\right.$

By the Uniform Agreement and Total Order properties of Total Order MBroadcast

F6.2 $\forall T \in \operatorname{submitted}(\rho), \forall o \in T, \forall i \in \operatorname{correct}(\rho),\left(\exists t\right.$, oop $\left.\left(T, G_{i, t}\right)\right) \Rightarrow\left(\forall T^{\prime}\right.$ submitted $(\rho), T^{\prime} \rightarrow T \Rightarrow$ $\left.\exists t,\left(T, T^{\prime}\right) \in G_{i, t}\right)$

Since $o \in o p\left(T, G_{i, t}\right)$ and $G_{i, 0}=(\varnothing, \varnothing)$, either:

1. $i \in \operatorname{replicas}(T) \wedge$ TO-deliver ${ }_{o}(i)$

First observe that since links are reliable $i \in$ replicas $(o)$.

Let $T^{\prime}$ be a a transaction, $o^{\prime} \in T^{\prime}$ an operation, and $k$ a site such that $o^{\prime} \rightarrow{ }_{k} O$.

By fact $\mathbf{F 6 . 1}$ since $i, j \in$ replicas $(o), o^{\prime} \rightarrow_{i} o$.

2. $\exists G \in \mathbb{G}$, receive $_{T}(G) \wedge o \in o p(T, G)$

According to Algorithm 2 it exists $k_{0}, \ldots, k_{m}$ sites sucht that:

- $k_{0}$ TO-delivers $o$ during $\rho$, and execute line 29 sending $\operatorname{pred}\left(T, G_{k_{0}}\right)$ with $o \in o p\left(T\right.$, predecessors $\left.T G_{k_{0}}\right)$ and $k_{1} \in \operatorname{replicas}\left(\right.$ out $\left(T, G_{k_{0}}\right)$ ).

- $k_{1}$ receives $\operatorname{pred}\left(T, G_{k_{0}}\right)$ during $\rho$ and then execute line 29 or line 34 sending a precedence graph $G$ such that $\operatorname{pred}\left(T, G_{k_{0}}\right) \subseteq G$ to a set of replicas containinig $k_{2}$.

. etc ... until $i$ receives it.

Consequently $\operatorname{pred}\left(T, G_{k_{0}}\right) \subseteq G_{i, t}$, and according to our reasonning in item 1, we conclude that fact $\mathbf{F 6 . 2}$ is true.

Fact F6.2 and proposition P5 conclude.

We are now able to proove our central theorem: every transaction is eventually closed at a correct site. 
$\forall T \in \operatorname{submitted}(\rho), \forall i \in \operatorname{correct}(\rho)$,

$$
\left(\exists t, T \in G_{i, t}\right) \Rightarrow\left(\exists t, \operatorname{closed}\left(T, G_{i, t}\right)\right)
$$

\section{Proof}

We consider that a finite subset of $\mathbb{T}$ are sent to the system, consequently submitted $(\rho)$ is also finite. Let $C_{T}$ be the graph resulting from the transitive closure of the relation $\rightarrow$ on $\{T\}$. According to proposition P6, $C_{T}$ is eventually in $G_{i, t}$, and thus according to proposition P5, $T$ is eventually closed at site $i$.

\section{P7}

$\forall T \in \operatorname{submitted}(\rho), \forall i, j \in \Pi, \forall t, t^{\prime}$, $\left(T \in G_{i, t} \wedge T \in G_{j, t^{\prime}} \wedge \operatorname{closed}\left(T, G_{i, t}\right) \wedge \operatorname{closed}\left(T, G_{j, t^{\prime}}\right)\right) \Rightarrow\left(\operatorname{pred}\left(T, G_{i, t}\right)=\operatorname{pred}\left(T, G_{j, t^{\prime}}\right)\right)$

Proof

$\mathbf{F 7 . 1} \operatorname{pred}\left(T, G_{i}\right) \cdot \mathcal{V}=\operatorname{pred}\left(T, G_{j}\right) \cdot \mathcal{V}$

Let $T^{\prime} \in \operatorname{pred}\left(T, G_{i}\right)$. By definition it exists $T_{1}, \ldots, T_{m}$ such that $T^{\prime} \rightarrow T_{1} \rightarrow$ $\ldots \rightarrow T_{m} \rightarrow T \subseteq G_{i}$. By an obious induction on $m$ using proposition $\mathbf{P 6}$ we conclude that $T^{\prime}$ is also in $\operatorname{pred}\left(T, G_{j}\right)$.

$\mathbf{F 7 . 2} \operatorname{pred}\left(T, G_{i}\right) \cdot \mathcal{E}=\operatorname{pred}\left(T, G_{j}\right) \cdot \mathcal{E}$

Identical to the reasonning proposed for fact F7.1.

F7.2 $\forall T^{\prime} \in \operatorname{pred}\left(T, G_{i}\right), o p\left(T^{\prime}, \operatorname{pred}\left(T, G_{i}\right)\right)=\operatorname{op}\left(T^{\prime}, \operatorname{pred}\left(T, G_{j}\right)\right)$

By fact F7.1 and since $T$ is closed at both sites $i$ and $j$.

F7.4 $\left\{T^{\prime} \mid\right.$ isAborted $\left.\left(T, G_{i}\right)\right\}=\left\{T^{\prime} \mid\right.$ isAborted $(T$, pgraphSitej $\left.)\right\}$

Let $T^{\prime} \in \operatorname{pred}\left(T, G_{i}\right)$ such that $i s A b o r t e d\left(T^{\prime}, \operatorname{pred}\left(T, G_{i}\right)\right)$. According to Algorithm 2, it exists a site $k$ and a read operation $r \in T^{\prime}$ such that $k$ TO-delivers $r$ during $\rho$, and then $k$ set the aborted flag of $T^{\prime}$ in its precedence graph.

Now let $k^{\prime}$ be a replica of $r$, by the Uniform Agreement and the Total Order Property of Total Order Multicast, when $k^{\prime}$ TO-delivers $r$, it also set the aborted flag of $T^{\prime}$ in its precedence graph. 
By the conjunction of facts F7.1 to F7.4.

We proove now that $\rho$ is serializable [2].

Let $O(x, \rho)$ be the set of write operation over the data item $x$ during $\rho$, we define the relation $\ll$ as follows:

$$
\forall x \in \mathbb{D}, \forall o_{1}, o_{2} \in O(x, \rho), x_{1} \ll x_{2} \triangleq \exists i \in \operatorname{replicas}(x), o \rightarrow_{i} o^{\prime}
$$

$\underline{\mathbf{P 8}} \ll$ is a version order for $\rho$.

\section{Proof}

Let $O(x, \rho)$ be the set of write operation over the data item $x$ during $\rho$; and let $i \in$ replicas $(x) \cap \operatorname{correct}(\rho)$ (assumption $\mathbf{A} \mathbf{1})$.

According to Algorithm 2$] o$ is executed only if $\operatorname{trans}(o)$ is committed during $\rho$ consequently $i$ commits during $\rho$ any transaction $T$ such that $\exists o \in w o(T), O(x, \rho)$. Consequently $\ll$ is total over $O(x, \rho)$, and by the Total Order and Uniform Agreement properties of Total Order Multicast, $\ll$ is an order over $O(x, \rho)$.

\section{P9}

$\forall T, T^{\prime} \in M V S G(\rho, \ll)$,

$$
\left(\left(T, T^{\prime}\right) \in \operatorname{MVSG}(\rho, \ll) \wedge w o(T) \neq \varnothing \wedge w o\left(T^{\prime}\right) \neq \varnothing\right) \Rightarrow T \rightarrow T^{\prime}
$$

Proof

F9.1 If $\left(T, T^{\prime}\right)$ is a read-from edge, then $T \rightarrow T^{\prime}$

Let $\left(T, T^{\prime}\right)$ be a read-from relation. By definition it exists a site $i$, a write $w[x] \in T$, and a read $r[x] \in T^{\prime}$ such that during $\rho$ at site $i w$ write $x$ then $r$ reads the value written by $w$.

Let $t$ and $t^{\prime}$ be respectively the times at which these two events occured; according to Algorithm 2]:

F9.1.1 TO-deliver $_{i}(o)<_{\rho} t<_{\rho} t^{\prime}$ 
Then since $T^{\prime} \in \operatorname{MVSG}(\rho, \rightarrow), T^{\prime} \in \operatorname{submitted}(r)$, and by assumption A1, it exists

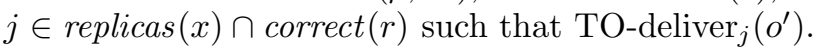

Now, TO-deliver $i(o) \Rightarrow$ TO-deliver $_{j}(o)$ by the Uniform Agreement, and the Total Order properties of Total Order Multicast. Consequently using fact F9.1.1,

$\neg\left(\right.$ TO-deliver $_{i}\left(o^{\prime}\right)<_{\rho}$ TO-deliver $\left._{i}(o)\right) \Rightarrow$ TO-deliver $_{j}(o)<_{\rho}$ TO-deliver $_{j}\left(o^{\prime}\right)$

concluding our claim.

F9.2 If $(T, T)$ is a version-order edge, then $T \rightarrow T$

Let $T_{1}, T_{2}, T_{3}$ be three transactions committed during $\rho$, and suppose that it exists a version-order edge $\left(T_{1}, T_{2}\right) \in \operatorname{MVSG}(\rho, \rightarrow)$.

According to the definition of a version order it follows either:

1. it exists $w_{1} \in w o\left(T_{1}\right), w_{2} \in w o\left(T_{2}\right)$, and $r_{3} \in \operatorname{ro}\left(T_{3}\right)$ such that $r_{3}\left[x_{3}\right], w_{1}\left[x_{1}\right]$ and $x_{1} \ll x_{2}$.

By definition of $x_{1} \ll x_{2} \Rightarrow T_{1} \rightarrow T_{2}$.

2. it exists $r_{1} \in \operatorname{ro}\left(T_{1}\right), w_{2} \in w o\left(T_{2}\right)$ and $w_{3} \in w o\left(T_{3}\right)$ such that $r_{1}\left[x_{3}\right], w_{2}\left[x_{2}\right]$ and $x_{3} \ll x_{2}$.

Let $i \in \operatorname{replicas}(x) \cap \operatorname{correct}(\rho)$ ( by assumption A1). Since $T_{1}, T_{2}, T_{3} \in \operatorname{committed}(r) \subseteq$ submitted $(r), i$ TO-delivers $r_{1}, w_{1}$ and $w_{3}$ during $\rho$. Now according to the Total Order property of Total Order Multicast, since $x_{3} \ll x_{2}, w_{3} \rightarrow{ }_{i} w_{2}$.

Let $j$ be a site on which $r_{1}[x 3]$ happens. Since $w_{3} \rightarrow_{i} w_{2}$, according to our definition of commit() (Section 3.8), $w_{2} \prec r_{1}$.

Now since $T_{1} \in \operatorname{committed}(\rho)$, necessarily $r_{1} \rightarrow_{i} w_{2}$ (otherwise $T_{1}$ is aborted: line 24).

By facts F9.1 and F9.2

P10 $\rho$ is serializable.

Proof

Consider the sub-graph $G_{u}$ of $M V S G(r, \ll)$ containing all the transactions $T$ such that $w o(T) \neq \varnothing$, and the edge linking them.

F10.1 $G_{u}$ is acyclic. 
Let $T_{1}, \ldots, T_{m} \in G_{u}$ such that $T_{1}, \ldots, T_{m \geq 1}$ forms a cycle in $G_{u}$, and recall that by definition $T_{1}, \ldots, T_{m} \in \operatorname{committed}(r)$

According to proposition P9, $T_{1} \rightarrow \ldots \rightarrow T_{m} \rightarrow T_{1}$.

Let $i$ be a replica of $T_{1}$, and we note $t$ the time at which $i$ commits $T$ during $\rho$. Acoording to Algorithm 2 at time $t, \operatorname{closed}\left(T_{1}, G_{i, t}\right)$.

Now according to Algorithm 1 , and since $i$ commits $T_{1}$ at time $t$,

$$
\exists k \in \llbracket 2, m \rrbracket, T_{k} \in \operatorname{breakCycles}\left(\operatorname{pred}\left(T_{1}, G_{i, t}\right)\right)
$$

Let $j \in \operatorname{replicas}\left(T_{k}\right)$ such that $j$ commit $T_{k}$ during $\rho$, and let $t$ " be the time at which this event happens.

Since $T_{1} \in \operatorname{pred}\left(T_{k}, G_{j, t^{\prime}}\right)$ and $T_{k} \in \operatorname{pred}\left(T_{1}, G_{i, t}\right), \operatorname{pred}\left(T_{1}, G_{i, t}\right)=\operatorname{pred}\left(T_{k}, G_{j, t^{\prime}},.\right)$ Consequently since breakCycles() is deterministic, $j$ cannot commit $T_{k}$ during $\rho$. Absurd.

F10.2 $\operatorname{MVSG}(\rho, \rightarrow)$ is acyclic.

By fact F10.1 and since read only transactions are executed using two-phases locking.

Fact F10.2 induces that $\rho$ is serializable. 
Unité de recherche INRIA Rocquencourt Domaine de Voluceau - Rocquencourt - BP 105 - 78153 Le Chesnay Cedex (France)

Unité de recherche INRIA Futurs : Parc Club Orsay Université - ZAC des Vignes 4, rue Jacques Monod - 91893 ORSAY Cedex (France)

Unité de recherche INRIA Lorraine : LORIA, Technopôle de Nancy-Brabois - Campus scientifique 615, rue du Jardin Botanique - BP 101 - 54602 Villers-lès-Nancy Cedex (France)

Unité de recherche INRIA Rennes : IRISA, Campus universitaire de Beaulieu - 35042 Rennes Cedex (France)

Unité de recherche INRIA Rhône-Alpes : 655, avenue de l'Europe - 38334 Montbonnot Saint-Ismier (France)

Unité de recherche INRIA Sophia Antipolis : 2004, route des Lucioles - BP 93 - 06902 Sophia Antipolis Cedex (France) 